\title{
Stochastic Reactive Power Compensation Using Capacitor Allocation Based on Modified Harmony Search Algorithm
}

\author{
Shyh-peng Wáng1, Yann Cheong; Orville Chén \\ Electrical and Computer Enginnering, \\ Yeungnam College of Science and Technology \\ Daegu, South Korea
}

\begin{abstract}
This paper presents a heuristics based approach for capacitor allocation problem incorporating Point Estimate Method (PEM) based analysis to attain optimal capacitor reallocation in radial distribution systems. The proposed probabilistic method is used to achieve optimal capacitor allocation associated with the active/reactive loads forecasted error as well as energy loss reduction by taking into account the cost function coefficients. This problem is formulated as a mix integer non-linear, discrete multi-objective problem which be in need of a robust optimization tool to avoid trapping in local optima. The proposed solution based on modified harmony search (MHS) algorithm employ to search for a set of Pareto optimal value of objective functions which save in memory that called repository. Moreover, a fuzzy clustering method is utilized to control the size memory (repository) for the concerned problem. The proposed approach has been tested on IEEE 9 buses to check feasibility and robustness of the proposed approach.
\end{abstract}

Keywords: Capacitor Allocation, Point Estimate Method (PEM), Fuzzy Membership Function, Self-Adaptive Modified Harmony Search Optimization (SAMHSO), Pareto Management.

\section{INTRODUCTION}

Capacitor bank are attached to radial distribution network to improve power factor and voltage profile as well as reduce loss, simultaneously. To attain these mentioned benefits under different operating constraints, the optimal locations, types, and size of shunt capacitor are required. However, multimodal nature, discrete limitations due to capacitor operation in discrete steps, non-linear characteristics of capacitor allocation problem arise challenges particularly for large-scale problem and as a result the number of points to be analyzed highly increase in the power networks.

In the past decades, outstanding research has been investigated into the capacitor bank allocation problem to solve the problem. The early proposed approaches generally based on dynamic programing techniques and analytical methods jointed with heuristics. Methods explored for optimal capacitor allocation can be categorized in details to analytical $[1,2]$ and heuristics search methods $[3,4]$, numerical programing approaches [5], and artificial intelligence (AI) based methods [6-9]. In [10], fuzzy logic theory idea is used to investigate the optimal capacitor allocation problem. Recently, fuzzy set theory has caught the attraction of researcher for designing intelligent systems in broad range of applications such as electrical load forecasting [11], designing fuzzy controller for industrial robots [12,13], powerful tool for system uncertainty compensator and optimization method with high rate of accuracy and performance [14,15], adaptive model for non-linear controllers [16,17], etc. Here the system is modeled by using fuzzy membership function which most challenging issue would be the proper choose of membership functions and identify corresponding rule properly and precisely.

A new simulated annealing technique in conjunction with fuzzy logic theory is proposed in [18] to attain the best solution on stable and robust manner. In recent years, the evolutionary methods inspired from the biological phenomena have been provided for handling and addressing complex optimization problem requirements. In [19], a two steps modified flower pollination optimization algorithm (FPOA) is presented to reach better performance in order to decrease the operating cost and consequently increase the annual net saving. A bacteria foraging algorithm depends upon on fuzzy logic theory [20] is proposed to look into search space and find the optimum solution for location of capacitors.

In [21] the genetic algorithm (GA) is utilized to search for optimum size and locations of capacitors in the distribution network and it evaluates through sensitivity analysis associated with GA. In this work, the suggested optimal solution relies on the appropriate selecting and initializing the adjusting probability parameters. Meanwhile, some new fast sensitivity-based heuristics associated with GA are introduced in $[22,23]$ which offered a better fulfillment than each of the methods separately. A particle swarm optimization (PSO) based approach [24] is utilized to search the optimal solution at various load levels in the radial system. Although, both GA and PSO algorithms have attain proper results in different optimization condition, their algorithm dependencies to initial tunable parameters and probability stuck in local optima points or premature convergence diminish effectiveness of the algorithms. On the other hand, when all aforementioned approaches show the good capability for finding the optimum solution of the capacitor allocation problem, the main shortage of them is abandoning impact of the uncertain parameters. The stochasticity of some variables such as active/reactive loads' forecasted error or even the cost coefficient variations can dispossess the networks from realistic optimization analysis.

Thus, a new probabilistic framework depends upon $2 \mathrm{~m}$ point estimate method (PEM) is proposed in this paper to investigate the impact of the uncertain parameters on the optimal capacitor allocation problem. Moreover, a new elf adaptive modification technique depends upon Harmony 
Search (HS) algorithm is proposed to find a Pareto solution of the stochastic multi-objective capacitor allocation problem. In order to bring the objective functions values in the same base compared to the huge difference between their values, the fuzzy set theory take into account to increase the performance of proposed method. Thus, among all solutions, the founded non-dominated solutions during search process will stack in an external memory (repository) and a clustering technique based on fuzzy membership function is performed to avoid enlarging the repository size.

\section{CAPACITOR ALLOCATION PROBLEM FORMULATION}

In this section, the capacitor allocation problem objective functions and practical equality and inequality constraints are formulated for realistic modeling of the system in order to define full considerations of different load levels, practical aspect of tie and close switched capacitor banks.

\subsection{Objective functions}

For a realistic definition of the system with respect to presence of no-linearity characteristic of loads, the cost function (per year) is consist of two main parameters such as the network total power and energy losses as well as the capacitor installation cost as formulated in (1) and (2) [25,26].

$\min f_{1}=\min (\cos t)$

$$
f_{1}(X)=\cos t(X)=K_{p} \times P_{T, \text { loss }}+\sum_{i=1}^{N_{b}} K_{i}^{c} \times Q_{i}^{c} \quad ; i=1, \ldots, N_{b}
$$

Where $X$ is decision vector which is defined in (3) in detail. $K_{p}$ and $K^{c} i$ stand for the equivalent cost per unit of power loss and the annual capacitor installation cost, respectively. The total power losses of the network is presented by $P_{T, l o s s}$. The amount of reactive power which compensated in $i^{\text {th }}$ bus defined by $\mathrm{Q}^{\mathrm{C}}{ }_{\mathrm{i}}$.

$X=\left[T_{1}, T_{2}, \ldots, T_{N_{b}}, Q_{1}^{c}, Q_{2}^{c}, \ldots ., Q_{N_{b}}{ }^{c}\right]_{1 \times\left(2 N_{b}\right)}$

Where $T i$ that corresponds to the two discrete values; 0 and 1 . It takes zero value when $i^{\text {th }}$ bus is not a suitable for compensation otherwise takes value 1 as a result $\mathrm{kVAr}$ (the reactive power) can applied to the $\mathrm{i}^{\text {th }}$ bus.

Traditional methods for the feeder power losses is determined through (4) [27].

$$
f_{2}(X)=p_{\text {loss }}(X)=\sum_{i=1}^{N_{b r}} R_{i} \times\left|I_{i}\right|^{2}
$$

In which $R i$ and $I i$ are resistance and actual current of the $\mathrm{i}^{\text {th }}$ branch.

The magnitude of voltage deviation is another objective function which need to follow (5) [28].

$$
f_{3}(X)=d_{\text {volt }}(X)=\max \left\{\left|1-V_{\min }\right|,\left|1-V_{\max }\right|\right\}
$$

\subsection{Practical constrains}

All limitation and operational constraints which must preserved through analysis for meet the practical conditions are determined and described as follow.

\subsubsection{Capacitor sizes}

The size of capacitors is discrete value related to the smallest capacitor size. From economic point of view, the large capacitors size imposes lower cost than the small size one. Consequently, the available capacitor bank size can be described in (6).

$Q_{M \mathrm{ax}}{ }^{c}=L \times Q_{0}{ }^{c}$

In which $Q^{c} 0$ denote the smallest size of the capacitors in $\mathrm{kVAr}$ that multiply an integer $(L)$. The available capacitor

\begin{tabular}{|c|c|c|c|c|}
\hline$i$ & 1 & 2 & 3 & 4 \\
\hline $\begin{array}{c}\text { Qc } \\
(k \vee A r)\end{array}$ & 150 & 300 & 450 & 600 \\
\hline$\$ / k V A r$ & 0.500 & 0.350 & 0.253 & 0.220 \\
\hline$i$ & 5 & 6 & 7 & 8 \\
\hline $\begin{array}{c}\text { Qc } \\
(\mathrm{kVAr})\end{array}$ & 750 & 900 & 1050 & 1200 \\
\hline$\$ / k V A r$ & 0.276 & 0.183 & 0.228 & 0.170 \\
\hline$i$ & 9 & 10 & 11 & 12 \\
\hline $\begin{array}{c}\text { Qc } \\
(k \vee A r)\end{array}$ & 1350 & 1500 & 1650 & 1800 \\
\hline$\$ / k V A r$ & 0.207 & 0.201 & 0.193 & 0.187 \\
\hline$i$ & 13 & 14 & 15 & 16 \\
\hline $\begin{array}{c}\text { Qc } \\
(\mathrm{kVAr})\end{array}$ & 1950 & 2100 & 2250 & 2400 \\
\hline$\$ / k V A r$ & 0.211 & 0.176 & 0.197 & 0.170 \\
\hline$i$ & 17 & 18 & 19 & 20 \\
\hline $\begin{array}{c}\text { Qc } \\
(k \vee A r)\end{array}$ & 2550 & 2700 & 2850 & 3000 \\
\hline$\$ / \mathrm{kVAr}$ & 0.189 & 0.187 & 0.183 & 0.180 \\
\hline$i$ & 21 & 22 & 23 & 24 \\
\hline $\begin{array}{c}\text { Qc } \\
(k \vee A r)\end{array}$ & 3150 & 3300 & 3450 & 3600 \\
\hline$\$ / \mathrm{kVAr}$ & 0.195 & 0.174 & 0.188 & 0.170 \\
\hline$i$ & 25 & 26 & 27 & \\
\hline $\begin{array}{c}\text { Qc } \\
(k \vee A r)\end{array}$ & 3750 & 3900 & 4050 & \\
\hline$\$ / k V A r$ & 0.183 & 0.182 & 0.179 & \\
\hline
\end{tabular}
sizes and the correspond cost are summarized in Table 1.

Table 1. The available capacitor sizes and cost

2.2.2 Distribution Line Capacity

The power flow over the feeders should follow the below constraint to guarantee the safe flow among buses.

$\left|P_{i j}^{\text {Line }}\right|<P_{i j, \text { max }}^{\text {Line }}$ 
Where $\left|P^{\text {Line }} i j\right|$ as an absolute power flow must be less than maximum power flow over the distribution line between two nodes of $i$ and $j$.

\subsubsection{Bus voltage limitation}

There is a limitation for the voltage level of ith bus to maintain the operating voltage range between the minimum and maximum constraints (Vmin, Vmax) as is formulated in (8).

$$
V_{\min } \leq V_{i} \leq V_{\max }
$$

\section{2m PEM STOCHASTIC ANALYSIS}

The optimal capacitor allocation problem for radial distribution networks incorporates several uncertain parameters which an appropriate probabilistic analyzer should utilize to consider intrinsic random characteristic of the problem variables. From the viewpoint of technical mathematics, the 2m PEM account as a more accurate stochastic method compare to analytical techniques. Despite the simulation based methods (Monte Carlo Simulation) the proposed stochastic analyzer based on $2 \mathrm{~m}$ PEM show less processing time for converging to optimum point. The main characteristics behind approximate methods such as $2 \mathrm{~m}$ PEM would be its appropriate capability for handling the uncertainties in the search space. As a positive point of $2 \mathrm{~m}$ PEM can points out to less need to data (the mean, variance, skewness, and etc.) from the probability function of the uncertain variables. In order to describe the mathematics rule of the $2 \mathrm{~m}$ PEM, it is simpler to formulate the deterministic power flow function as a non-linear function as shown in (9).

$$
S=F(z)
$$

In which $z$ is defined as an input vector related to load, branch data, and the topology of network. Correspondingly, $S$ output vector consists the information about line power flow, and voltage profile is perspective of the uncertainty in the input vector $z$. In other word, $2 m$ PEM runs power flow function to approximate the output vector $S$ moments. According Eq. (9) a few number of moments of $z$ are needed in which for $l^{\text {th }}$ input variable of $z$ vector, a $f_{z l}$ (probability distribution function) is defined as shown in (10) Error! Reference source not found. The initial values of probability point's is selected by $f_{z l}$ matching with two points through mean, variance, and skewness coefficient according $2 m$ PEM.

$$
S=F\left(\mu_{z 1}, \mu_{z 2}, \ldots z_{l, k}, \ldots, \mu_{z m}\right) ; k=1,2
$$

Where $z l, k$ is kth location point of the random variable $z_{l}$. Two variables $z l, 1$ and $z l, 2$ stand for two random variables location of $z l$ which is defined in (11). Also, the standard location of the random variables $z_{l}\left(\zeta_{l, k}\right)$ is selected as follow:

$z_{l, k}=\mu_{z_{l}}+\xi_{l, k} \cdot \sigma_{z_{l}} ; \quad k=1,2$

$$
\xi_{l, k}=\frac{\lambda_{l, 3}}{2}+(-1)^{3-k} \sqrt{m-\left(\lambda_{l, 3}^{2} / 2\right)^{2}}, \quad k=1,2
$$

Where $\mu_{z j}$ and $\sigma_{z l}$ defined as mean value of the $j^{\text {th }}$ random variable and standard deviation of the random variable $z_{l}$, respectively. Also, $\lambda_{l, 3}$ stand for the skewness coefficient. The $2 m$ PEM transferred the space variables in the locations $z l, 1$ and $z l, 2$ to the output variables $S_{l, l}$ and $S_{l, 2}$ by utilizing weight parameters. In this case, two weight parameters are used to map $z l, 1$ and $z l, 2$ on the output data set. Finally, the third central moment $\left(\lambda_{l, 3}\right)$ is defined based on expected operator $(E)$ as follow:

$\lambda_{l, 3}=\frac{E\left[\left(z_{l}-\mu_{z_{l}}\right)^{3}\right]}{\left(\sigma_{z_{l}}\right)^{3}}$

Accordingly, the standard deviation value $(\sigma)$ of $S_{i}$ is indicated by variance of output vector (var) as below.

$\sigma=\sqrt{\operatorname{var}\left(S_{i}\right)}=\sqrt{E\left(S_{i}^{2}\right)-\left[E\left(S_{i}\right)\right]^{2}}$

\section{MULTI-OBJECTIVE OPTIMIZATION ALGORITHM}

As stated before, the capacitor allocation optimization problem is a type of discrete, complex, and nonlinear integer programming associated with multiple objective functions which some of these fitness functions correlated to each other. Thus, the powerful optimization tool will require to cope with all non-linearity and Pareto problems. This paper proposed a self-adaptive modified harmony search (MHS) algorithm to address the deficiencies of the previous methods for handling the conflicting targets of the problem. Mathematically, the concerned multi-objective optimization problem is formulated in (15) incorporate to the constraints [30].

$$
\begin{aligned}
& \min F=\left[f_{1}(X), f_{2}(X), \ldots, f_{n}(X)\right]^{T} \\
& h_{i}(X)=0 \quad i=1,2, \ldots, N_{e q} \\
& g_{i}(X)<0 \quad i=1,2, \ldots, N_{\text {ueq }}
\end{aligned}
$$

Moreover, two main below conditions must be satisfied in terms of multi-objective minimization problem in which the $\mathrm{X} 1$ solution determine as a dominant solution if both (16) and (17) are satisfied as below.

$$
\begin{aligned}
& \forall j \in\{1,2, \ldots, n\}, f_{j}\left(X_{1}\right) \leq f_{j}\left(X_{2}\right) \\
& \exists k \in\{1,2, \ldots, n\}, f_{k}\left(X_{1}\right)<f_{k}\left(X_{2}\right)
\end{aligned}
$$


In the case of not satisfying any of the two above conditions, then this results not selecting the $\mathrm{X} 1$ solution as dominated one compared to $\mathrm{X} 2$ so it counts as non-dominated solutions.

Generally, in a given fuzzy system, linguistic variables are defined to model the system behavior as word or sentence compared to the mathematical models which is defined as numerical values. For a given engineering problem, linguistic variables might be different depends upon the area of problem. For example, fuzzy logic controller is one of the most well-known application of fuzzy logic in which linguistic variables can be defined as error and change of error [31]. The complete set of optimization process data is stored in repository which its size needs to be controlled by fuzzybased clustering technique. There are several fuzzy membership functions available in the literature namely linear, Gaussian, singleton, triangular, trapezoidal. Here, fuzzy membership function offers the capability to maintain all value associate with the concerned objective functions in the same base in a way that repository size keeps constant. In this case, a fuzzy membership function for each one of the objectives functions is dedicated as formulated in (18). For each one of the Pareto optimal solution sorted in repository, a normalized membership function is evaluated in (19) based on available decision options.

$$
\begin{gathered}
M_{i}(X)=\left\{\begin{array}{cc}
1 & \text { for } f_{i}(X) \leq f_{i}^{\min } \\
0 & \text { for } f_{i}(X) \geq f_{i}^{\max } \\
\frac{f_{i}^{\max }-f_{i}(X)}{f_{i}^{\max }-f_{i}^{\min }} f_{i}^{\min } \leq f_{i}(X) \leq f_{i}^{\max }
\end{array}\right. \\
N_{M F}(j)=\frac{\sum_{i=1}^{n} \Delta_{i} \times M F_{i}\left(X_{j}\right)}{\sum_{j=1}^{N p} \sum_{i=1}^{n} \Delta_{i} \times M F_{i}\left(X_{j}\right)}
\end{gathered}
$$

Where $f_{i}^{\min }, f_{i}^{\max }$ are defined as minimum and maximum boundaries of the $i^{\text {th }}$ objective function (fi(X)). In (19), $\mathrm{MF}_{\mathrm{i}}(\mathrm{X})$ is described as fuzzy membership function for the $\mathrm{i}^{\text {th }}$ objective function. The fuzzy membership function determines as an adaptive decision making criteria which can change based on available decision candidate. All nondominated solutions are evaluated by NMF and at the end the best non-dominated solution will save in the repository.

\section{SELF-ADAPTIVE MODIFIED HARMONY SEARCH (SAMHS)}

\subsection{Original harmony search algorithm}

Harmony search mimics by process of musician search for finding an ideal harmony. It has a tight relationship with the composing process of each harmony. Each harmony is perspective of one solution for concerning optimization problem. The main steps of the harmony search (HS) algorithm are summarized as follow Error! Reference source not found.:
Step 1- Parameters initialization: The setting parameters of the algorithm such as decision variables, upper/lower boundaries of each variable, harmony memory (HM) size, harmony memory consideration rate (HMCR), pitch adjusting rate (PAR), and bandwidth vector (BW) are initialized in this step.

Step 2- Harmony memory (HM) initialization: A random matrix for $\mathrm{HM}$ is generated in which each component of this matrix is initialized using uniform random number between upper/lower boundaries of the decision variables.

Step 3- New harmony improvisation: Generally, there are three main rules which are utilized for new harmony improvisation. Each note in $\mathrm{HM}$ is played by a musician to get to the highest harmony based on three subsets that are listed in below:

- Harmony memory consideration: Each component of the new harmony vector is selected from stored corresponding components in the HM randomly. This selection is performed based on HMCR formulated in (20) as the selecting probability which is determined between 0 and 1 .

$$
x_{k h}^{n e w}=\left\{\begin{array}{lc}
x_{k h}^{H M} & \text { rand } \prec H M C R \\
x_{k h}^{\text {rand }} & \text { otherwise. }
\end{array}\right.
$$

- Pitch adjustment: The selected components from HM modify depends upon PAR and BW which stand for the probability of a selected for modification component from $\mathrm{HM}$ and distance bandwidth, respectively. Wherein the BW parameter as the bandwidth is updated in each iteration $(i)$ as follows:

$$
\begin{aligned}
& x_{k h}^{\text {new }}= \begin{cases}x_{k h}^{H M} \pm \text { rand } \times B W & ; \\
x_{k h}^{\text {rand }} & ; \text { rand }<P A R\end{cases} \\
& B W(i)=B W_{\max } \times e^{\rho i} \\
& \rho=\operatorname{Ln}\left(\frac{B W_{\min }}{B W_{\max }}\right) \times N I^{-1}
\end{aligned}
$$

In this step, each new produced note checks to assure that it does not need more tune. In other word, after performing step 1 and initializing parameters such $H M, B W$, and $P A R$ an internal updating step need to adopt $P A R$ varying in regard to search capability improvement by total number of improvisation stages $(N I)$. Thus, updating $P A R$ value dynamically is computed in (24).

$$
P A R(i)=P A R_{\min }+\frac{i}{N I} \times\left(P A R_{\max }-P A R_{\min }\right)
$$

Where the pitch adjustment rate is defined as $P A R(i)$ in generation $I$ at population. Also, $P A R \max$ and $P A R$ min stand for maximum and mini-mum tolerance of adjustment rate. 
- Random generation: A random selection among each not selected components from memory consideration is performed between upper/lower bounds. As a result, the generation probability of random component would be (1-HMCR).

- Harmony memory update: The new improved harmony is assessed based on the objective function in a way that if the objective function value of new harmony vector is better than its peer of worst harmony in the $\mathrm{HM}$ then the current value of harmony is replaced with the new one. After maximum possible number of improvisation is reached, the process stopped and the best harmony (solution) is store in memory. If the stopping criterion does not meet step 3 and 4 are repeated.

\subsection{Self-adaptive technique}

Sub-modification

In the original HS algorithm, setting small PAR value along with large value of BW increase computational burden needed for exploring the optimal solution as a result deteriorate performance of optimization tool. Therefore, the original HS method needs some modification to improve its performance for multi-objective problem particularly. In this paper a modification stages are explained to improve the improvisation steps beside increase the optimization algorithm convergence capability depends upon the adaptive modified HS algorithm. The proposed self-adaptive technique is divided into two sub-modifications which each one can be adaptively selected according to their necessities during the optimization process.

\subsubsection{Sub-modification1:}

As stated above, in each iteration ( $i$ ) of the original HS a fourstep procedure is applied to create and update new harmony. Clearly, this procedure can be merge with genetic operators, i.e., crossover and mutation in order to improve HS performance. In the original HS, the new produced notes are generated as formulated in (25).

$$
X_{M u t, 1}=X_{n 1}+\Im_{1} \times\left(X_{n 2}-X_{n 3}\right)
$$

Where three notes, $X_{n 1}, X_{n 2}$, and $X_{n 3}$ are selected from population in random such that $n_{1} \neq n_{2} \neq n_{3}$. By using the mutation operator. Then, by using crossover operator three new notes are generated as follow:

$$
\begin{aligned}
& \left.x_{\left.n n 1,2 N_{b}\right)}\right] ; \overline{X_{n, 2}}=\left[x_{n n 2,1}, x_{n n 2,2}, \ldots, x_{\left.n n 2,2 N_{b}\right)}\right] \\
& x_{n n 1, i}= \begin{cases}x_{m u t, i}, & \text { if } \gamma_{1} \leq \gamma_{2} \\
x_{n o t e, ~}, & \text { otherwise }\end{cases} \\
& x_{n n 2, i}= \begin{cases}x_{m u t, i}, & \text { if } \gamma_{2} \leq \gamma_{3} \\
x_{i}, & \text { otherwise }\end{cases}
\end{aligned}
$$

Now the best note is selected based on modified step for the new improved harmony. The modification step will increase the diversity of the harmony population, sufficiently.

\subsubsection{Sub-modification2:}

In the second modification step, value of $\mathfrak{I}_{\text {update based on }}$ (27). The adaptive formulation of $\mathfrak{I}$ update is described after several consecutive running process.

$$
\mathfrak{J}^{k+1}=(1 / 3 \text { Iter })^{1 / \text { ter }} \mathfrak{J}^{k}
$$

Each sub-modification steps will determine by specific probability to be chosen correctly. First, both submodifications determine with the same probability $\left(P_{\theta}=0.33\right.$; $\theta=1,2)$. Then, accumulator (A) is defined to recognize the probability of each sub-modification which is assigned initially zero. Next, $X_{1}$ and $X_{N p o p}$ as the best and worst solutions in the harmony population will sort according to their fitness function in the descending order. Finally, for each harmony will be allocated a weighting factor $(W)$ as shown in (28).

$$
W_{j}=\frac{\log \left(N_{p o p}-j+1\right)}{\log (1)+\ldots+\log \left(N_{p o p}\right)} j=1, \ldots, N_{p o p}
$$

Now, all the accumulators are updated through (29).

$$
A_{\theta}=A_{\theta}+\frac{W g t_{l}}{n_{\operatorname{Mod}_{\theta}}} l=1, \ldots, n_{M^{\prime} d_{\theta}}
$$

In the above equation, $\eta_{\text {Mod } \theta}$ shows the number of notes which have chosen $\theta^{\text {th }}$ sub-modification step. In addition, accumulator value will determine the probability of submodification methods in each iteration (Iter) as follow:

$$
P_{\theta}=(1-\nabla) \times P_{\theta}+\nabla \times \frac{A_{\theta}}{\text { Iter }},(\theta=1,2,3)
$$

Where $\nabla$ stand for the learning speed which equals $\omega=0.142$, experimentally. Finally, the normalized probability of each modification method is evaluated in (31).

$$
P_{\theta}=P_{\theta} /\left(\sum_{\theta=1}^{3} P_{\theta}\right)
$$

For each iteration, each harmony will choose $\theta^{\text {th }}$ submodification methods according their probabilities.

\section{SOLUTION PROCEDURE}

The sequence of steps for applying SAMHSO algorithm to minimize/maximize optimal objective functions of the problem, are given through flowchart below: 


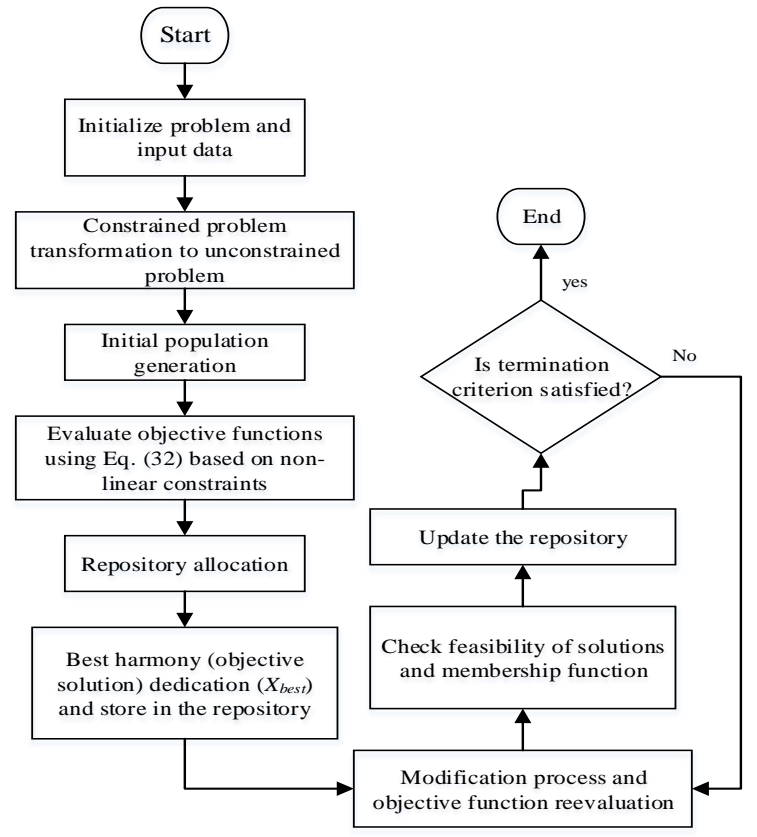

Figure. 1 Block diagram of SAMHSO algorithm by the proposed method

The proposed optimization method with self-adaptive modification techniques is implemented for the multiobjective capacitor allocation optimization problem. First, all economic data, optimization constraints, termination criterion, and input date of optimization tool, and objective functions are defined then constrained problem is transformed to unconstrained.

In the next step, the non-dominated solution result of the first phase of objective function evaluation are stored in the repository and recheck its size. Then, the best solutions are chosen depends upon their constraints. Next, the population is improved by the sub-modification steps to improve intensification and diversification of optimization process. After modifying the population, the objective functions reevaluate and feasibility of them are checked. If the best individual dominates the $i^{\text {th }}$ harmony, the harmony will be replaced by that individual else it will remain in its position. Thus, the repository is updated based on the new places and then termination criterion is checked. If criteria satisfied finish the algorithm, otherwise, go to modification step and repeat the process.

\section{SIMULATION}

In this section, the concerned optimization problem is solved utilizing a notional distribution network Error! Reference source not found.. Fig. 2 shows the single-line diagram of the test system which is investigated as the case study. The case study is IEEE 9-bus test system with radial structure supply one feeder with the line voltage $23 \mathrm{kV}$.

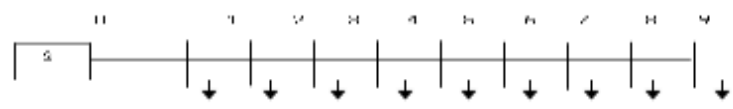

Figure. 2 IEEE 9-bus distribution test system

The network data details of case study can be found in Error! Reference source not found. regard feeder topology and load characteristics. The nominal active and reactive load values are $12368 \mathrm{~kW}$ and $4186 \mathrm{kVAr}$, respectively. The simulation results divided to two phases, deterministic and stochastic analysis. In the first part, the proposed SAMHSO algorithm is applied to solve capacitor allocation for each of three objective functions (loss, and voltage deviation and annual price). Fig. 3 are shown to show the investigated objective functions for 9 buses system. Also, Table. 2 depicted to obtain the capacitor size and corresponding locations of the test system based on SAMHSO algorithm.

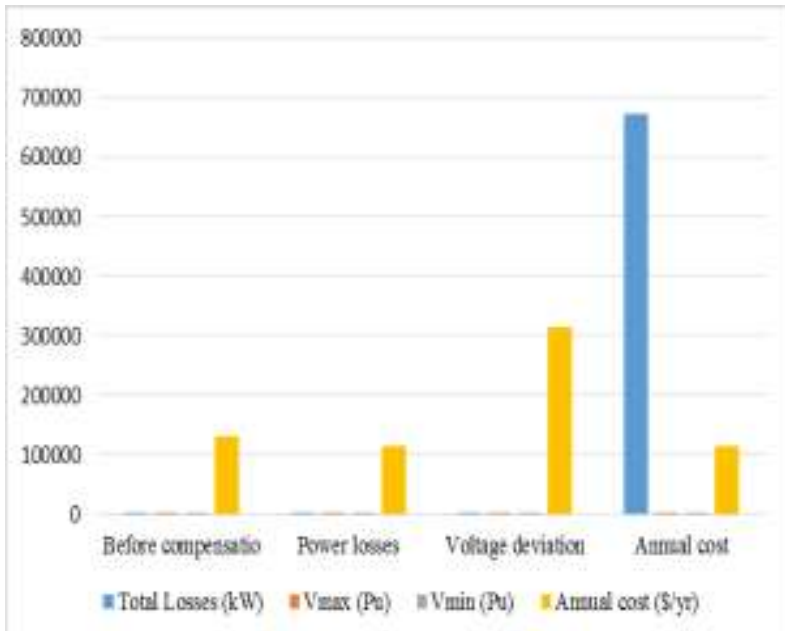

Figure. 3. Deterministic analysis of single objective function depends upon the proposed SAMHSO

As it can be seen from Fig.3, the objective functions' values effectively decreased by using the proposed SAMHSO algorithm compare to the case before compensation. The annual price and active power losses are shown same behavior in the single optimization process. Despite these both cases, the voltage deviation is high compare to the base case without compensation. By defining the voltage deviation as the single objective function can attain the minimum value of voltage deviation in range of $0.00501(\mathrm{Pu})$ which is a satisfying value. However, the power losses and annual price are increased. Therefore, all objective functions must be improving effectively in the case of single objective optimization problem.

Table 2. Capacitor location and size through deterministic analysis

\begin{tabular}{|c|c|c|c|c|c|c|c|c|}
\hline & \multicolumn{8}{|c|}{ Capacitor location (bus number) } \\
\hline Bus No. & 1 & 2 & 3 & 4 & 5 & 6 & 7 & 9 \\
\hline $\begin{array}{c}\text { Optimum } \\
\text { Power } \\
\text { losses }\end{array}$ & 150 & 4050 & 1350 & 2100 & 900 & 150 & 300 & 300 \\
\hline Bus No. & 5 & 7 & 8 & & 9 & & & \\
\hline $\begin{array}{c}\text { Optimum } \\
\text { Voltage } \\
\text { deviation }\end{array}$ & 600 & 4050 & 1950 & & 3450 & & & \\
\hline Bus No. & 2 & 3 & 4 & & 5 & 7 & & 9 \\
\hline $\begin{array}{c}\text { Optimum } \\
\text { Annual } \\
\text { cost }\end{array}$ & 3300 & 900 & 2100 & & 900 & 450 & & 300 \\
\hline
\end{tabular}


For comparing the robustness of the proposed algorithm with the well-known optimization algorithms Error! Reference source not found. which are recently investigated by the researchers, Fig.4, 5, and 6 are depicted for capacitor allocation problem in the distribution network. The multiobjective problem by using the SAMHSO algorithm is applied to case study by the bus voltage limits in the range of $[0.9,1.1]$

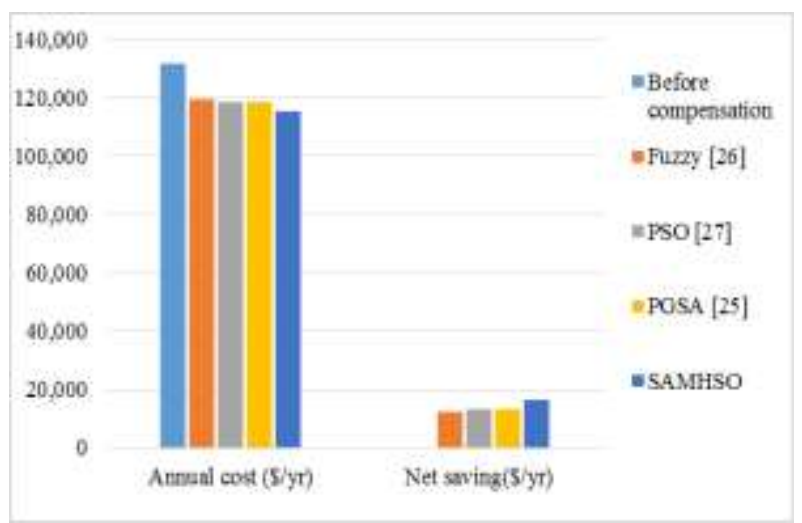

$\mathrm{Pu}$.

Figure. 4. Deterministic analysis of single objective function depends upon the proposed SAMHSO

Figure. 5. Comparison of deterministic analysis of capacitor allocation by different algorithms for loss reduction and saving percent

Figure. 6. Comparison of deterministic analysis of capacitor allocation by different algorithms for Total loss $(\mathrm{kW})$

As it can be seen from above figures (4, 5, and 6), the proposed optimization method (SAMHSO) shows better performance in finding the pareto solution than the other wellknown methods Error! Reference source not found.. Thus, the performance of SAMHSO algorithm should assay in

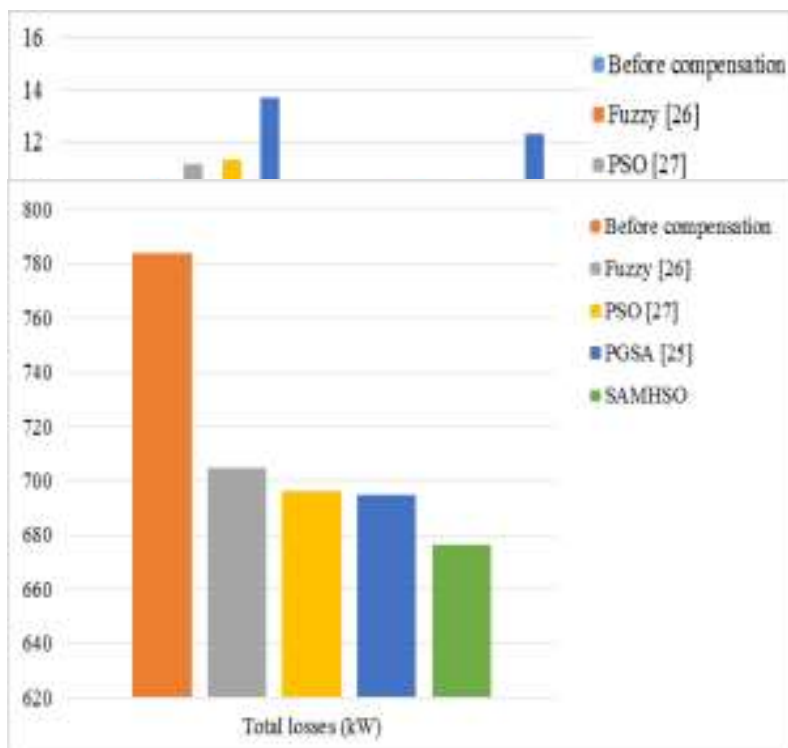

stochastic analysis. In order to involve the probabilistic analyzer to the capacitor allocation problem, the $2 \mathrm{mPEM}$ is consider into the problem. Result are shown in Fig.7 describe the best optimum values for multi-objective functions for IEEE 9 buses case study. For the sake of better and precise comparison, the results compared with the deterministic framework

(a)

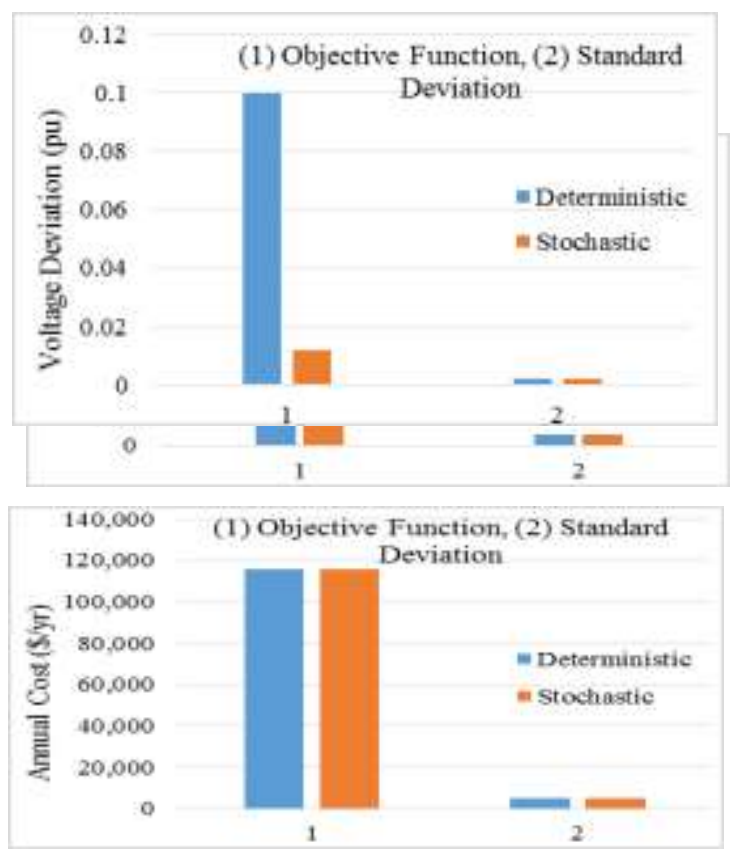

(b)

(c)

Figure. 7. Comparison of deterministic and stochastic analysis of capacitor allocation of multi-objective optimization

The stochastic analysis cost is shown into incremental growth of the optimal values of the objective functions. In fact, the uncertain parameters impose some non-linearity into the problem which by considering the uncertainties the optimal values are increased. However, considering the uncertainties offers more realistic analysis for the real applications. As it can be seen from results reported in Fig.7, the proposed probabilistic method has diminished the standard deviation values of the multi-objective functions. It is noteworthy to note that the proposed approach with probabilistic analysis still shows better performance compared to the other common used methods.

\section{CONCLUSION}

Like several heuristics methods such as PSO, GA, and GSA for solving the capacitor allocation into distribution network a need for a novel optimization algorithm would be necessary which be capable to cope with high volume of non-linearity in the problem. In this paper an efficient and precise stochastic framework is considered depends upon SAMHSO algorithm associated with $2 \mathrm{~m}$ PEM. The proposed self-adaptive submodification techniques is consisted of two different modification method. Optimal capacitor allocation is solved 
by SAMHSO algorithm and its capability in solving nonconvex problem will increase the convergence ability of method. The proposed approach has been tested on IEEE 9 buses case study with superior results regarding the fast convergence and benefits of networks compared to most common used methods. Moreover, effectiveness and feasibility of the proposed method was shown through multiobjective functions optimization problem.

\section{REFERENCES}

[1] Das, D. "Optimal placement of capacitors in radial distribution system using a Fuzzy-GA method." International Journal of Electrical Power \& Energy Systems 30.6 (2008): 361-367.

[2] B. Papari, C. S. Edrington, T. Vu, "Stochastic Operation of Interconnected Microgrids", IEEE Power and Energy Society (PES), 2017, Chicago, USA.

[3] Khakpour, A., D. Asemani, and B. Papari. "Adaptive Noise Cancellation based Architecture for Correction of Gain and Offset Mismatch Errors in Time-Interleaved ADC." Iranian Journal of Science and Technology. Transactions of Electrical Engineering 38.E2 (2014): 149.

[4] C. F Chang, Reconfiguration and Capacitor Placement for Loss Reduction of Distribution Systems by Ant Colony Search Algorithm, IEEE Trans. Power. Syst, 23(4) (2008) 1747-1755.

[5] Papari, B., D. Asemani, and A. Khakpour. "A wide-band time-interleaved A/D converter for cognitive radio application with adaptive offset correction." Wireless Advanced (WiAd), 2011. IEEE, 2011.

[6] R. Baldick, F.F. Wu, Efficient integer optimization algorithms for optimal coordination of capacitors and regulators, IEEE Trans. Power Syst. 5 (1990) 805-812.

[7] Vu, T. V., Perkins, D., Papari, B., Vahedi, H., \& Edrington, C. S. (2017, June). Distributed adaptive control design for cluster of converters in DC distribution systems. In DC Microgrids (ICDCM), 2017 IEEE Second International Conference on (pp. 197-201). IEEE.

[8] Jabr, R. A. "Optimal placement of capacitors in a radial network using conic and mixed integer linear programming." Electric Power Systems Research 78.6 (2008): 941-948.

[9] Vu, T., Gonsoulin, D., Perkins, D., Papari B., Vahedi, H., Edrington, C. S. (2017, August). Distributed Control Imple-mentation for Zonal MVDC Ship Power Systems. In Electric Ship Technologies Symposium (ESTS), 2017 IEEE (pp. 14-18).

[10] Olamaei, J., M. Moradi, and T. Kaboodi. "A new adaptive modified firefly algorithm to solve optimal capacitor placement problem." Electrical Power Distribution Networks (EPDC), 2013 18th Conference on. IEEE, 2013.

[11] Al-Kandari, A.M., Soliman, S.A. and El-Hawary, M.E., 2004. Fuzzy short-term electric load forecasting. International Journal of Electrical Power \& Energy Systems, 26(2), pp.111-122.

[12] G. Sahamijoo, O. Avatefipour, M. R. S. Nasrabad, M. Taghavi, F. Piltan, Research on minimum intelligent unit for flexible robot,International Journal of Advanced
Science and Technology, Vol. 80,No. 6, pp. 79-104, 2015.

[13] Mokhtar, M., Piltan, F., Mirshekari, M., Khalilian, A., \& Avatefipour, O. (2014). Design minimum rule-base fuzzy inference nonlinear controller for second order nonlinear system. International Journal of Intelligent Systems and Applications, 6(7), 79.

[14] Khalilian, A, Piltan, F, Avatefipour, O, Safaei MR, Sahamijoo, G. Design New Online Tuning Intelligent Chattering Free Fuzzy Compensator, International Journal of Intelligent Systems and Applications(IJISA), vol.6, no.9, pp.75-86, 2014. DOI: 10.5815/ijisa.2014.09.10

[15] Shahcheraghi, A., Piltan, F., Mokhtar, M., Avatefipour, O., \& Khalilian, A. (2014). Design a Novel SISO Offline Tuning of Modified PID Fuzzy Sliding Mode Controller. International Journal of Information Technology and Computer Science (IJITCS), 6(2), 72.

[16] Park, J.H., Seo, S.J. and Park, G.T., 2003. Robust adaptive fuzzy controller for nonlinear system using estimation of bounds for approximation errors. Fuzzy Sets and Systems, 133(1), pp.19-36.

[17] Avatefipour, O., Piltan, F., Nasrabad, M. R. S., Sahamijoo, G., \& Khalilian, A. (2014). Design New Robust Self Tuning Fuzzy Backstopping Methodology. International Journal of Information Engineering and Electronic Business, 6(1), 49.

[18] B Papari, CS Edrington, F Kavousi-Fard, “An Effective Fuzzy Feature Selection and Prediction Method for Modeling Tidal Current: A Case of Persian Gulf", IEEE Trans. Geoscience and Remote Sensing, vol. 99, 2017, pp. 1-6.

[19] Bhattacharya S.K, S.K. Goswami , A new fuzzy based solution of the capacitor placement problem in radial distribution system, Elzevier Expert Systems with Applications 36 (2009) 4207-4212.

[20] Abdelaziz, A. Y., E. S. Ali, and SM Abd Elazim. "Optimal sizing and locations of capacitors in radial distribution systems via flower pollination optimization algorithm and power loss index." Engineering Science and Technology, an International Journal 19.1 (2016): 610-618.

[21] Tabatabaei, S. M., and B. Vahidi. "Bacterial foraging solution based fuzzy logic decision for optimal capacitor allocation in radial distribution system." Electric Power Systems Research 81.4 (2011): 1045-1050.

[22] Papari. B., Asemany, D., Khakpour, A., "An Adaptive Mismatch Error Cancellation Architecture for TimeInterleaved A/D Converters." International a journal of Electronics and Communication Technology (IJECT); vol. 4, Spl. 5, 2013, pp. 9-13.

[23] Grainger, John J., and S. H. Lee. "Optimum size and location of shunt capacitors for reduction of losses on distribution feeders." IEEE Transactions on Power Apparatus and Systems 3 (1981): 1105-1118.

[24] Huang, Shyh-Jier. "An immune-based optimization method to capacitor placement in a radial distribution system." IEEE Transactions on Power Delivery 15.2 (2000): 744-749.

[25] Lee, Kang Seok, and Zong Woo Geem. "A new metaheuristic algorithm for continuous engineering optimization: harmony search theory and practice." 
Computer methods in applied mechanics and engineering 194.36 (2005): 3902-3933.

[26] R. Srinivasa Rao, S. V. L. Narasimham, Optimal Capacitor Placement in a Radial Distribution System using Plant Growth Simulation Algorithm, World Academy of Science, Engineering and Technology 45 (2008) 715-722.

[27] C. T. Su, C. C. Tsai, A new fuzzy reasoning approach to optimum capacitor allocation for primary distribution systems, Proc IEEE Indus. Tech.Conf. (1996) 237-41.

[28] B Papari, CS Edrington, I bhattacharya, G Radman, "Effective Energy Management of Hybrid AC-DC Microgrids with Storage Devices", IEEE Trans. Smart Grid, vol. 99, 2017, pp. 1-6.

[29] Yu, Xin-mei, Xin-yin Xiong, and Yao-wu Wu. "A PSObased approach to optimal capacitor placement with harmonic distortion consideration." Electric Power Systems Research 71.1 (2004): 27-33.

[30] El-Fergany, Attia A., and Almoataz Y. Abdelaziz. "Artificial bee colony algorithm to allocate fixed and switched static shunt capacitors in radial distribution networks." Electric Power Components and Systems 42.5 (2014): 427-438

[31] Papari, B., C. S. Edrington, T. V. Vu, and F. DiazFranco. "A heuristic method for optimal energy management of DC microgrid." In DC Microgrids (ICDCM), 2017 IEEE Second International Conference on, pp. 337-343. IEEE, 2017.

[32] Olamaei, J., M. Moradi, and T. Kaboodi. "A new adaptive modified firefly algorithm to solve optimal capacitor placement problem." Electrical Power Distribution Networks (EPDC), 2013 18th Conference on. IEEE, 2013.

[33] Anderson, Adam L., C. Brett Witherspoon, and B. Papari. "Spectrum recognition in large-scale cognitive radio networks with spectral data mining." Proceedings of WORLDCOMP (2014).

[34] Khalilian, A., Sahamijoo, G., Avatefipour, O., Piltan, F., \& Nasrabad, M. R. S. (2014). Design high efficiencyminimum rule base PID like fuzzy computed torque controller. International Journal of Information Technology and Computer Science (IJITCS), 6(7), 77.

[35] Lin, Chi-Ming, and Mitsuo Gen. "Multi-criteria human resource allocation for solving multistage combinatorial optimization problems using multiobjective hybrid genetic algorithm." Expert Systems with Applications 34.4 (2008): 2480-2490.

[36] T. Niknam, A. Kavousifard, S. Tabatabaei, J. Aghaei, Optimal operation management of fuel cell/wind/photovoltaic power sources connected to distribution networks, J. Power Sources 196 (2011) 8881- 8896. 